\title{
Multi-label classification via multi-target regression on data streams
}

\author{
Aljaž Osojnik $^{1,2}$ (I) Panče Panov ${ }^{1} \cdot$ Sašo Džeroski $^{1,2,3}$
}

Received: 26 April 2016/ Accepted: 18 November 2016 / Published online: 30 December 2016

(C) The Author(s) 2016. This article is published with open access at Springerlink.com

\begin{abstract}
Multi-label classification (MLC) tasks are encountered more and more frequently in machine learning applications. While MLC methods exist for the classical batch setting, only a few methods are available for streaming setting. In this paper, we propose a new methodology for MLC via multi-target regression in a streaming setting. Moreover, we develop a streaming multi-target regressor iSOUP-Tree that uses this approach. We experimentally compare two variants of the iSOUP-Tree method (building regression and model trees), as well as ensembles of iSOUP-Trees with state-of-the-art tree and ensemble methods for MLC on data streams. We evaluate these methods on a variety of measures of predictive performance (appropriate for the MLC task). The ensembles of iSOUP-Trees perform significantly better on some of these measures, especially the ones based on label ranking, and are not significantly worse than the competitors on any of the remaining measures. We identify the thresholding problem for the task of MLC on data streams as a key issue that needs to be addressed in order to obtain even better results in terms of predictive performance.
\end{abstract}

Keywords Multi-label classification · Multi-target regression · Data stream mining

Editors: Nathalie Japkowicz and Stan Matwin.

Aljaž Osojnik

aljaz.osojnik@ijs.si

Panče Panov

pance.panov@ijs.si

Sašo Džeroski

saso.dzeroski@ijs.si

1 Jožef Stefan Institute, Jamova Cesta 39, Ljubljana, Slovenia

2 Jožef Stefan International Postgraduate School, Jamova Cesta 39, Ljubljana, Slovenia

3 Centre of Excellence for Integrated Approaches in Chemistry and Biology of Proteins, Jamova Cesta 39, Ljubljana, Slovenia 


\section{Introduction}

The task of multi-label classification (MLC) has recently become very prominent in the machine learning research community (Gibaja and Ventura 2015). It can be seen as a generalization of the ubiquitous multi-class classification task, where instead of a single label, each example is associated with multiple labels. This is one of the reasons why multi-label classification is the go-to approach when it comes to automatic annotation of media, such as images, texts or videos, with tags or genres. Most research into multi-label classification has been performed in the batch learning context. However, some effort has also been made to explore multi-label classification in the streaming setting (Qu et al. 2009; Read et al. 2012; Bifet et al. 2009), following the popularity of big data in the research community, as well as in industry. With an appropriate method, working in the streaming context allows for real-time analysis of large amounts of data, e.g., emails, blogs, RSS feeds, social networks, etc.

Due to the nature of the streaming setting, there are several constraints that need to be considered. A data stream is a potentially infinite sequence of examples, which needs to be analyzed with finite resources, in particular, in finite time and memory. The largest point of divergence from the batch setting is the fact that the underlying concept (that we are trying to learn) can change at any point in time. Therefore, algorithm design is often divided into two parts: (1) learning a stationary concept, and (2) detecting and adapting to its changes. In this paper, we propose a method for multi-label classification in the streaming context that focuses on learning the stationary concept (or more precisely, a set of concepts).

Many algorithms in the literature take the problem transformation approach to multi-label classification, both in the batch and the streaming setting (Read et al. 2008, 2011; Tsoumakas and Vlahavas 2007; Fürnkranz et al. 2008). They transform the multi-label classification problem into several problems that can be solved with the off-the-shelf methods, e.g., a transformation into an array of binary classification problems. With this transformation, the label inter-correlations can be lost, and, consequently, the predictive performance can decrease.

In this paper, we take a different perspective and transform the multi-label classification problem into a multi-target regression problem. Multi-target regression is a generalization of single-target regression, used simultaneously predict multiple continuous variables (Struyf and Džeroski 2006; Appice and Džeroski 2007). Many facets of multi-label classification are also present in multi-target regression, e.g., correlation between labels/variables, which motivated us to approach multi-label classification by using multi-target regression methods.

To address the multi-label classification task, we have developed a straightforward multilabel classification via multi-target regression methodology, and used it in combination with a streaming multi-target regressor (iSOUP-Tree). The generality is a strong point of this approach, as it allows us to address multiple types of structured output prediction problems, such as multi-label classification and hierarchical multi-label classification, in the streaming setting.

In our initial work on this topic (Osojnik et al. 2015), we performed a set of preliminary experiments with the aim to show that multi-label classification via multi-target regression is a viable approach. We compared our algorithms with basic MLC methods (that give as output a single classifier). We used a very limited number of evaluation measures.

In this paper, we introduce several novel aspects. First, we introduce an adaptive perceptron in the leaves of the iSOUP-Tree, instead of the simple perceptron used in the initial work. Furthermore, we introduce an ensemble method (bagging) that uses iSOUP-Trees as base level learners and compare it with the state-of-the-art ensemble method for MLC in a streaming setting. Finally, we significantly extend the experimental methodology and the 
experimental questions. In particular, we include a wide range of evaluation measures in the comparison of the different methods and assess whether the overall differences in performance across all employed methods are statistically significant (by employing appropriate statistical tests).

The structure of the paper is as follows. First, we present the background and related work (Sect. 2). Next, we present the approach of multi-label classification via multi-target regression on data streams (Sect. 3) and our iSOUP-Tree method for MTR on data streams (Sect. 4). Furthermore, we present the research questions and the experimental design (Sect. 5). We then present and discuss the results (Sect. 6). Finally, we outline our conclusions and some directions for further work (Sect. 7).

\section{Background and related work}

In this section, we review the state-of-the art in multi-label classification, both in the batch and the streaming context. In addition, we present the background of the multi-target regression task, which we use as a foundation for defining the multi-label classification via multi target regression approach.

\subsection{Multi-label classification}

Generalizing multi-class classification, where only one of the possible labels needs to be predicted, multi-label classification requires a model to predict a combination (subset) of the possible labels. Formally, this means that for each data instance $\mathbf{x}$ from an input space $X$ a model needs to provide a prediction $\hat{y}$ from an output space $Y$, which is a powerset of the labelset $\mathcal{L}$, i.e., $Y=2^{\mathcal{L}}$. This is in contrast to the multi-class classification task, where the output space is simply the labelset $Y=\mathcal{L}$. We denote the real labels of an instance $\mathbf{x}$ by $y$, and a prediction made by a model for $\mathbf{x}$ by $\hat{y}(\mathbf{x})$ (or simply $\hat{y}$ ).

In the batch setting, the problem transformation approach is commonly used to tackle the task of multi-label classification. Problem transformation methods are usually used as basic methods to compare to, and are used in a combination with off-the-shelf base algorithms. The most common approach, called binary relevance (BR), transforms a multi-label task into several binary classification tasks, one for each of the possible labels (Read et al. 2011). Binary relevance models have been often overlooked due to their inability to account for label correlations, though some BR methods are capable of modeling label correlations during classification.

Another common problem transformation approach is the label combination or label powerset (LC) method, where each subset of the labelset is considered as an atomic label for a multi-class classification problem (Read et al. 2008; Tsoumakas and Vlahavas 2007). If we start with a multi-label classification task with a labelset of $\mathcal{L}$, we transform this into a multi-class classification with a lableset $\mathcal{L}^{\prime}=2^{\mathcal{L}}$.

The third most common problem transformation approach is pairwise classification, where we have a binary model for each possible pair of labels (Fürnkranz et al. 2008). This method performs well in some contexts. For larger problems the method becomes intractable because of model complexity.

In addition to problem transformation methods, there are also adaptations of the well known algorithms that handle the task of multi-label classification directly. Examples of such algorithms are the adaptation of the decision tree learning algorithm for MLC (Vens et al. 2008), support-vector machines for MLC (Gonçalves et al. 2013), k-nearest neighbours 
for MLC (Zhang and Zhou 2005), instance based learning for MLC (Cheng and Hüllermeier 2009), and others.

\subsection{Multi-label classification on data streams}

Many of the problem transformation methods for multi-label classification have also been used in the streaming context. Unlike the batch context, where a fixed and complete dataset is given as input to a learning algorithm, the streaming context presents several limitations that the stream learning algorithm must take into account. Bifet and Gavaldà (2009) define the most relevant ones as follows: (1) the examples arrive sequentially; (2) there can potentially be infinitely many examples; (3) the distribution of examples need not be stationary; and (4) after an example is processed it is discarded or archived. The fact that the distribution of examples is not presumed to be stationary means that algorithms should be able to detect and adapt to changes in the distribution (concept drift).

The first approach to MLC in data streams was a batch-incremental method that trains stacked BR classifiers (Qu et al. 2009). Some methods for multi-class classification, such as Hoeffding Trees (HT) (Domingos and Hulten 2000), have also been adapted to the multi-label classification task (Read et al. 2012). Hoeffding trees are incremental anytime decision trees for learning from data streams that use the notion that a small sample is usually sufficient for choosing an optimal splitting attribute, i.e., the use of the Hoeffding bound. Read et al. (2012) proposed the use of multi-label Hoeffding trees with pruned sets (PS) at the leaves (HTPS), as well as using them in combination with the ADWIN bagging (Bifet et al. 2009) ensemble method, which implicitly addresses the problems of change detection and adaptation. Bifet et al. (2010) introduced the Java-based Massive Online Analysis (MOA) ${ }^{1}$ framework, which also allows for the analysis of concept drift (Bifet and Gavaldà 2009) and has become one of the main software frameworks for data stream mining.

Recently, Spyromitros-Xioufis (2011) introduced a parameterized windowing technique for dealing with concept drift in multi-label data in a data stream context. Next, Shi et al. (2014a) proposed an efficient and effective method to detect concept drift based on label grouping and entropy for multi-label data, where the labels are grouped by using clustering and association rules. This allowed for an effective detection of concept drift which takes into account label dependence. Finally, Shi et al. (2014b) proposed an efficient class incremental learning algorithm, which dynamically recognizes some new frequent label combinations.

\subsection{Multi-target regression}

In the same way as multi-label classification generalizes regular (single target) classification, multi-target regression task is an extension of single-target regression. Multi-target regression (MTR) is the task of predicting multiple numeric variables simultaneously. Formally, the task is to make a prediction $\hat{y}$ from $\mathbb{R}^{n}$, where $n$ is the number of targets for a given instance $\mathbf{x}$ from an input space $X$.

As in multi-label classification, there is a common problem transformation method that transforms the multi-target regression problem into multiple single-target regression problems. In this case, we consider each numeric target separately and train a single-target regressor for each of them. However, this local approach suffers from similar problems as the problem transformation approaches to multi-label classification: The single target models do not consider the inter-correlations of the target variables. The task of simultaneous prediction of all target variables at the same time (the global approach) has been considered

1 URL: http://moa.cms.waikato.ac.nz/, accessed on 2016/04/23. 
in the batch setting by Struyf and Džeroski (2006). In addition, Appice and Džeroski (2007) proposed an algorithm for stepwise induction of multi-target model trees. Finally, Xioufis et al. (2016) introduced two new methods for multi-target regression (called Stacked SingleTarget and Ensemble of Regressor Chains) by adapting multi-label classification methods. The methods treat the other prediction targets as additional input variables and exploit the target dependencies in order to improve the accuracy of their predictions.

In the streaming context, some work has been done on multi-target regression. Ikonomovska et al. (2011b) introduced an instance-incremental streaming tree-based singletarget regressor (FIMT-DD) that utilized the Hoeffding bound. This work was later extended to the multi-target regression setting (Ikonomovska et al. 2011a) (FIMT-MT). There has been a theoretical debate on whether the use of the Hoeffding bound is appropriate (Rutkowski et al. 2013), but, a recent study by Ikonomovska et al. (2015) has shown that, in practice, the use of the Hoeffding bound produces good results. However, the drawback of these algorithms is that they ignore nominal input attributes. Recently, Duarte and Gama (2015) implemented a rule-based learning approach for multi-target regression (AMRules), while Shaker and Hüllermeier (2012) introduced an instance-based system for classification and regression (IBLStreams), which can be used for multi-target regression.

\section{Multi-label classification via multi-target regression}

The problem transformation methods (see Sect. 2.1) generally transform a multi-lablel classification task into one, or several, binary or multi-class classification tasks. In this paper, we take a different approach and transform a classification task into a regression task. The simplest example of a transformation of this type is to transform a binary classification task into a regression task. For example, if we have a binary target with labels yes and no, we would consider a numeric target to which we would assign a numeric value of 0 if the binary label is no and 1 if the binary label is yes.

In the same way, we can approach the multi-class classification task. Specifically, if the multi-class target variable is ordinal, i.e., the class labels have a meaningful ordering, we can assign the numeric values from 0 to $n-1$ to each of the corresponding $n$ labels. This makes sense, since if the labels are ordered, a misclassification of a label into a "nearby" label is better than a misclassification into a "distant" label. However, if the variable is not ordinal this makes less sense, as any given label is not in a strict relationship with other labels.

In that case, an approach similar to that introduced by Frank et al. (1998) to address multi-class classification using regression can be used. In their case, they produced several versions of the observed data, one version per class in the multi-class classification task. For each class, its version of the data featured a derived binary classification target, which corresponded to the presence of the class. Consequently, for each class a model tree regressor was learned. For a given example, the prediction of each of the trees was calculated, after which the example was classified into the class with the highest corresponding (numeric) tree prediction. This approach produces one regressor per class, however, with the use of methods for multi-target regression, this can be reduced to one (multi-target) regressor for all of the classes.

To address the multi-label classification task using regression, we transform it into a multitarget regression task (see Fig. 1). This procedure is performed in two steps: first, we take the viewpoint that the multi-label classification target is composed of several binary classification variables, just as in the BR method. However, instead of training one classifier for each of 
MLC

MTR

$\begin{array}{cccc}\text { Target space } & y \subseteq \mathcal{L}\left\{\lambda_{1}, \ldots, \lambda_{n}\right\} & \stackrel{\text { transformation }}{\longrightarrow} & y \in \mathbb{R}^{n} \\ \text { Instance } & y=\left\{\lambda_{1}, \lambda_{3}, \lambda_{4}\right\} & \stackrel{\text { transformation }}{\longrightarrow} & y=(1,0,1,1, \ldots)\end{array}$

Fig. 1 Transformation of a MLC problem to a MTR problem. Only the target space is transformed. Applied before learning a multi-target regressor

\section{MTR}

Target space

Instance

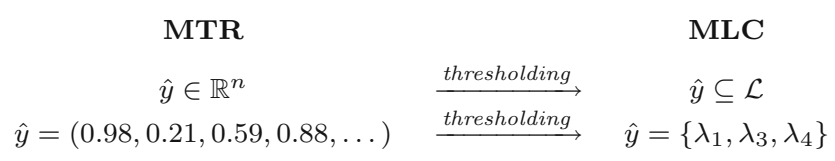

Fig. 2 From MTR to MLC. Transforming a multi-target regression prediction into a multi-label classification one

the binary variables, we further transform the values of the binary variable into numbers. A numeric target corresponding to a given label has a value 1 if the label is present in a given instance, and a value 0 if the label is not present.

For example, if we have a multi-label classification task with target labels $\mathcal{L}=$ \{red, blue, green\}, we transform it into a multi-target regression task with three numeric target variables $y^{\text {red }}, y^{\text {blue }}, y^{\text {green }} \in \mathbb{R}$. If an instance is labeled with red and green, but not blue, the corresponding numeric targets will have values $y^{\text {red }}=1, y^{\text {blue }}=0$, and $y^{\text {green }}=1$.

Since we are using a regressor, it is possible that a prediction for a given instance will not result in a value of exactly 0 or 1 for each of the targets. For this purpose, we use thresholding to transform back a multi-target regression prediction into a multi-label one (see Fig. 2). Namely, we construct the multi-label prediction in such a way that it contains labels with numeric values over a certain threshold, i.e., in our case, the labels selected are those with a numeric value over the threshold of $\tau=0.5$. It is clear, however, that a different choice of threshold leads to different predictions.

In the batch setting, thresholding can be performed in the pre- and postprocessing phases. However, in the streaming setting it needs to be done in real time. Specifically, the process of thresholding occurs at two times. The first thresholding occurs when the multi-target regressor has produced a multi-target prediction, which must then be converted into a multilabel prediction. The second thresholding occurs when we are updating the regressor, i.e., when the regressor is learning. Most streaming regressors are heavily dependent on the values of the target variables in the learning process, so the instances must be converted into the numeric representation that the multi-target regressor can utilize.

The problem of thresholding is not only problematic in the MLC via MTR setting, but also when considering the MLC task with other approaches. In general, MLC models produce results which are interpreted as probability estimations for each of the labels, thus the threhsolding problem is a fundamental part of multi-label classification.

\section{The iSOUP-Tree method}

To utilize the MLC via MTR approach, we have reimplemented the FIMT and FIMT-MT algorithms (Ikonomovska et al. 2011a) in the MOA framework to facilitate usability and visibility, as the original implementation was a standalone extension of the C-based VFML library (Hulten and Domingos 2003) and was not available as part of a larger data stream 
mining framework. We have also significantly extended the algorithm to consider nominal attributes in the input space when considering splitting decisions. This allows us to use the algorithm on a wider selection of datasets, some of which are considered herein.

In this paper, we combined the multi-label classification via multi-target regression methodology, proposed in the previous section, with the extended version of FIMT-MT, reimplemented in MOA. We named this method the incremental Structured OUtput Prediction Tree (iSOUP-Tree), since it is capable of addressing multiple structured output prediction tasks, i.e., multi-label classification and multi-target regression.

Ikonomovska et al. (2011b) have considered the performance of FIMT-DD when a simple predictive model is placed in each of the leaves, i.e., in this case a single linear unit (a perceptron). Model trees produce the predictions as a linear combination of input attribute values, i.e., $\hat{y}(\mathbf{x})=\sum_{i=1}^{m} x_{i} w_{i}+b$, where $m$ is the number of input attributes and $w_{i}, b$ are the perceptron weights, respectively. In contrast, in regression trees the prediction in a given leaf for an instance $\mathbf{x}$ is made for each of the targets as the average value of the recorded target values, $\hat{y}(\mathbf{x})=\frac{1}{|S|} \sum_{y \in S} y$, where $S$ is the set of observed examples in a given leaf. It was shown that using model trees yields better performance. However, this was only experimentally confirmed for regression tasks. In regression the targets generally exhibit larger variation than in classification tasks.

Our initial research showed that the use of a simple perceptron in the leaves provides very bad experimental results in the MLC via MTR setting (Osojnik et al. 2015). To correct this, we have replaced the perceptron with an adaptive perceptron, as done by Duarte and Gama (2014). This adaptive perceptron combines the predictions of the perceptron and the mean target predictor.

\subsection{Adaptive perceptron}

In the original implementation of FIMT by Ikonomovska et al. (2011b), the perceptron was always used to make the prediction. However, the adaptive model in a given tree leaf records the errors of the perceptron and compares them to the errors of the mean target predictor, which predicts the value of the target by computing the average value of the target over the examples observed in the leaf. In essence, each leaf has two predictors, the perceptron and the target mean predictor. The prediction of the predictor with the lower error (at a given point in time) is then used as the output prediction.

To monitor the errors, we use the faded mean absolute error which is calculated as

$$
f M A E_{\text {predictor }}(m)=\frac{\sum_{i=1}^{m} 0.95^{m-i}\left|\hat{y}_{i}-y_{i}\right|}{\sum_{i=1}^{m} 0.95^{m-i}},
$$

where $m$ is the number of observed examples in a leaf, $\hat{y}_{i}$ and $y_{i}$ are the predicted and real value for the $i$ th example, respectively, and predictor $\in\{$ perceptron, targetMean $\}$. The faded error is, in essence, weighted towards more recent examples. Intuitively, the numerator of the above fraction is the faded sum of absolute errors, while the denominator is the faded count of examples. For example, the most recent ( $m$ th) example contributes with a weight of 1 , the previous example with weight 0.95 , and the first example with weight $0.95^{m-1}$. This places a large emphasis on more recent examples and generally benefits the perceptron, as we expect its errors to decrease as it learns the weight vector.

However, we have to be careful when considering a classification task through the lens of regression. In classification, the actual target variables can only take values of 0 and 1 . If we use a linear model such as a perceptron (or the adaptive perceptron described above) to predict one of the targets, we have no guarantee that the predicted value will land in the $[0,1]$ interval. 
A regression tree's prediction will produce a prediction which is calculated as an average of zeroes and ones, which will always land in this interval. Additionally, the perceptrons in the leaves are trained in real-time according to the Widrow-Hoff rule, which consumes a non-negligible amount of time, which can be a constraint in the data stream mining setting. Hence, we are motivated to consider the use of both multi-target regression trees as well as multi-target model trees when addressing the task of multi-label classification via multitarget regression. We denote the regression tree variation of iSOUP-Tree as $i S O U P-R T$ and the model tree variant as $i S O U P-M T$.

\subsection{Ensembles}

In addition to observing and evaluating a single regression or model tree, we also consider ensembles of iSOUP-Trees. We use the online bagging approach introduced by Oza (2005), which naturally extends the approach for bagging from the batch setting. In essence, each of the incoming examples is assigned to each of the members of the ensemble a different number of times, i.e., for each example-ensemble member pair we sample the Poisson distribution with parameter $\lambda=1$ to determine the number of repetitions of the given example to the given ensemble member. The theoretical motivation behind this methodology is concisely explained in the original paper. We denote the bagging of iSOUP regression trees $E_{B} R T^{2}$ and the bagging of model trees as $E_{B} M T$.

Ensembles can also be used to address the problem of drift detection and adaptation. ADWIN bagging (Bifet et al. 2009) is an extension of the above ensemble methodology, which monitors the performance of the ensemble members and discards under-performing models, and replaces them with new empty models, which are learned anew. However, we specifically avoid the use of ADWIN bagging, as we wish to address the problem of change detection and adaptation even in the single-tree scenario.

\section{Experimental design}

In this section, we first present the experimental questions that we want to answer. Next, we describe the datasets and algorithms used in the experiments. Furthermore, we discuss the evaluation measures used in the experiments. Finally, we conclude with a description of the employed experimental methodology.

\subsection{Experimental questions}

Our experimental design is constructed in such a way to address several lines of inquiry. First, we investigate whether if the use of model trees with the adaptive perceptron improves predictive performance over regression trees. Namely, we have shown a previous study that using model trees with regular preceptrons produces considerably worse results than regression trees (Osojnik et al. 2015).

Second, we comparatively evaluate the performance of the introduced single tree methods to the Hoeffding tree with pruned sets $\left(H T_{P S}\right)$ (Read et al. 2012). The latter is a direct (single) tree-based competitor, which does not utilize the MLC via MTR methodology. This allows further investigates the viability of the proposed methodology for MLC.

${ }^{2} \mathrm{E}$ denotes that the method learns an ensemble, while the $\mathrm{B}$ determines that bagging is used to achieve variation among the base models. 
Table 1 Datasets used in the experiments

$N$ number of instances, $Q$ number of labels, $\phi_{L C}$ average number of labels per instance

\begin{tabular}{lrlll}
\hline Dataset & \multicolumn{1}{c}{$\mathrm{N}$} & Attribs. & $\mathrm{Q}$ & $\phi_{L C}$ \\
\hline 20NG & 19,300 & 1001 binary & 20 & 1.1 \\
Enron & 1,702 & 1001 binary & 53 & 3.4 \\
IMDB & 120,919 & 1001 binary & 28 & 2.0 \\
Ohsumed & 13,929 & 1002 binary & 23 & 1.7 \\
Slashdot & 3782 & 1079 binary & 22 & 1.2 \\
TMC & 28,596 & 500 binary & 22 & 2.2 \\
\hline
\end{tabular}

Furthermore, we compare all of the methods, including ensemble-based approaches, to determine how the methods rank both in terms of performance and efficiency, as well as to observe the effect of using ensembles of the base learners.

Finally, we observe the methods' efficiency to determine what, if any, trade-offs in terms of performance versus resource use are made when using the different methods.

\subsection{Datasets}

In our experiments, we use a subset of the datasets listed in Read et al. (2012, Tab.3) (see Table 1). Here, we briefly describe the dataset domains:

- The 20 newsgroups is a dataset comprised of a collection of articles from 20 newsgroups.

- The Enron dataset (Read 2008) is a collection of labelled emails, which, though small by data stream standards, exhibits some data stream properties, such as time-order and evolution over time.

- The $I M D B$ dataset is constructed from text summaries of movie plots from the Internet Movie Database and is labelled with the relevant genres.

- The Ohsumed dataset was constructed from a collection of peer-reviewed medical articles and labelled with the appropriate disease categories.

- The dataset Slashdot was collected from the http://slashdot.org web page and consists of article blurbs labelled with subject categories.

- The TMC dataset was used in the SIAM 2007 Text Mining Competition and consists of human generated aviation safety reports, labelled with the problems being described (we are using the version of the dataset specified in Tsoumakas and Vlahavas (2007)).

With the exception of the TMC dataset, all datasets are available at the MEKA project page. ${ }^{3}$ The TMC dataset is available at the Mulan data repository. ${ }^{4}$

\subsection{Algorithms}

To address our experimental questions, we performed experiments using our implementations of the algorithms for learning multi-target model trees (iSOUP-MT or MT for brevity) and multi-target regression trees ( $i S O U P-R T$ or $R T$ ). In addition, we also use ensemble methods, specifically, online bagging for iSOUP-RT $\left(E_{B R T}\right)$ and iSOUP-MT $\left(E_{B M T}\right)$. The testing for splits occurs at intervals of 200 observed examples, with the Hoeffding bound confidence level (the $\delta$ parameter) set to 0.0000001 .

\footnotetext{
3 https://sourceforge.net/projects/meka/files/Datasets/, accessed on 2016/03/11.

4 http://mulan.sourceforge.net/datasets-mlc.html, accessed on 2015/05/25.
} 
The MLC setting has not received as much attention in the streaming setting as it has in the batch setting, therefore, there aren't as many competing algorithms as there would be in the batch setting. We chose the updated implementation ${ }^{5}$ of Read et al. (2012), which learns Hoeffding trees with pruned sets $\left(H T_{P S}\right)$, as well as ADWIN bagging for Hoeffding trees with pruned sets $\left(E_{A} H T_{P S}\right){ }^{6}$ The parameters of these methods were set as suggested by the authors.

\subsection{Evaluation measures}

In the evaluation, we use a set of measures used in recent surveys and experimental comparisons of different multi-label algorithms in the batch setting (Madjarov et al. 2012; Gibaja and Ventura 2015). The evaluation measures are grouped into four segments: example-based measures (accuracy, $\mathrm{F}^{1}$, Hamming score), label-based measures (macro precision, macro recall, macro $\mathrm{F}^{1}$, micro precision, micro recall, micro $\mathrm{F}^{1}$ ), ranking-based measures (average precision, ranking loss, logarithmic loss), and efficiency measures (memory consumption and time). This yields a total of 12 measures of predictive performance and 2 measures of efficiency.

From the above, it is clear that in the MLC setting performance along a wide variety of measures can be investigated. Example-based measures evaluate the quality of classification on a per-example basis, i.e., how good is the classification over different examples, while label-based measures evaluate the quality of the classification on a per-label basis, i.e., how good is the classification over different labels. Ranking-based measures evaluate the classification based on the ordering of the labels according to their presence, e.g., a classification is evaluated more positively if the present labels are ranked higher, often without regard for the thresholding procedure.

In particular, example-based and label-based measures are calculated based on the comparison of the predicted labels with the ground truth labels. On one hand, example-based measures depend on the average difference of the actual and predicted sets of labels over the complete set of data examples from the evaluation set. On the other hand, label-based measures assess the performance for each label separately and than average the performance over all labels. The models produced by algorithms used in this study give as prediction numerical values for each of the labels. The label is predicted as present if the numerical value exceeds a predefined threshold $\tau$ (in our case we set the value to 0.5 ). This means that both example-based and label-based measures are directly dependent on the choice of the parameter $\tau$. Ranking-based evaluation measures, however, compare the predicted ranking of the labels with the ground truth ranking and do not necessarily depend on the choice of the threshold parameter. The full definitions of the observed measures can be found in "Appendix".

To measure the efficiency of the observed methods we consider the running time, measured in seconds, with a resolution of one hundredth of a second, and the total amount of memory consumed in MB. The time measurements exclusively measure the learning time and the time used to make predictions, excluding other processes such as loading of examples from the file system and the calculation of evaluation measures. In the case of time and memory usage, we desire low values.

\footnotetext{
5 The methods are implemented as part of the MEKA and MOA frameworks.

6 As before, E denotes the use of an ensemble, while the A stands for ADWIN bagging.
} 
Each evaluation measure presents and choosing which to optimize in a real-world scenario is dependent on the desired outcomes. The performance of competing methods is, therefore, evaluated separately using each measure. However, note that ranking-based measures are of special importance, as they do not require threhsholding, while precision and recall can be traded off by selecting a different threshold.

\subsection{Experimental setup}

For all of our experiments we are using the predictive sequential (prequential) evaluation methodology for data streams (Gama 2010). This means that for each example, first a prediction is made and collected, and second, the example is used to update the model. Once predictions for each of the examples are collected, the evaluation measures are calculated on all of the predictions. Using prequential evaluation ensures that the model has as much information as possible to make the prediction for each example. However, the prequential evaluation methodology is more optimistic than the other commonly used holdout evaluation approach, where a window of examples is constructed and the entire window is first used to make predictions and then to update the model.

Unlike the holdout methodology, the prequential evaluation methodology allows the model to use all of the information available at a given point to make a prediction, as all of the preceding examples are used to update the model prior to making a prediction. While in real-world applications either evaluation methodology could be the correct choice, in this paper, we chose to observe the performance of the methods in the most optimistic scenario.

More specifically, we constructed the following experimental setup to answer the proposed experimental questions. This experimental setup is designed to be a streaming analog of the commonly used batch MLC experimental setup, e.g., used by Madjarov et al. (2012) and Read et al. (2009), and is very similar to the setup used by Read et al. (2012) in the streaming setting.

For each of the datasets, we used the prequential methodology to calculate the predictions of all of the models on all of the instances in the dataset. The predictions are then thresholded to calculate the label-based and example-based measures on the entire dataset, while the ranking measures are calculated using the unthresholded predictions. The recorded measurements are therefore calculated using the obtained predictions over the entire dataset. Additionally, we measured the time and memory used to learn and make predictions.

To assess whether the overall differences in performance across all employed methods are statistically significant for a given evaluation measure, we employed the corrected Friedman test (Friedman 1940) and the post-hoc Nemenyi test (Nemenyi 1963) as recommended by Demšar (2006). The results of the statistical test are represented in the form of average rank diagrams for each evaluation measure. These form the basis on which we build the answers to our experimental questions and form our conclusions. When comparing only two methods, i.e., in the case of the comparison of regression and model trees as well as the comparison of different single-tree methods, we also refer to the results on the individual datasets.

\section{Results and discussion}

The results of the evaluation are grouped by the type of evaluation measure for ease of discussion. Within each group of evaluation measures, we discuss their relevance to our experimental questions. Afterwards, we wrap up with a discussion of the implication of the complete set of results to the experimental questions. 
Table 2 Predictive performance results: example-based measures

\begin{tabular}{|c|c|c|c|c|c|c|}
\hline & $M T$ & $R T$ & $E_{B R T}$ & $E_{B M T}$ & $H T_{P S}$ & $E_{A} H T_{P S}$ \\
\hline \multicolumn{7}{|l|}{ (a) Accuracy } \\
\hline $20 \mathrm{NG}$ & $0.1142(4)$ & $0.1174(3)$ & $0.0682(5)$ & $0.0648(6)$ & $0.3182(1)$ & $0.2773(2)$ \\
\hline Enron & $0.2438(1)$ & 0.1797 (4) & $0.1887(3)$ & $0.2379(2)$ & $0.0022(5)$ & $0.0010(6)$ \\
\hline IMDB & $0.0187(3)$ & $0.0026(5)$ & 0.0007 (6) & $0.0031(4)$ & $0.0435(2)$ & 0.1955 (1) \\
\hline Ohsumed & $0.1563(4)$ & $0.1611(3)$ & $0.1143(5)$ & $0.1035(6)$ & $0.3178(1)$ & $0.2980(2)$ \\
\hline Slashdot & $0.0049(3)$ & $0.0003(4)$ & $0.0000(6)$ & $0.0003(4)$ & $0.1393(2)$ & $0.1452(1)$ \\
\hline TMC & $0.3448(2)$ & $0.3479(1)$ & $0.3439(3)$ & $0.3317(4)$ & $0.0112(5)$ & $0.0094(6)$ \\
\hline Avg. rank & 2.83 & 3.33 & 4.67 & 4.33 & 2.67 & 3.00 \\
\hline \multicolumn{7}{|l|}{ (b) $\mathrm{F}_{e x}^{1}$} \\
\hline $20 N G$ & $0.1146(4)$ & 0.1177 (3) & $0.0683(5)$ & $0.0649(6)$ & $0.3205(1)$ & $0.2804(2)$ \\
\hline Enron & $0.3296(1)$ & $0.2411(4)$ & $0.2530(3)$ & $0.3221(2)$ & $0.0039(5)$ & $0.0015(6)$ \\
\hline IMDB & $0.0227(3)$ & $0.0031(5)$ & $0.0008(6)$ & 0.0037 (4) & $0.0597(2)$ & $0.2469(1)$ \\
\hline Ohsumed & $0.1767(4)$ & $0.1829(3)$ & $0.1280(5)$ & $0.1156(6)$ & $0.3612(1)$ & $0.3382(2)$ \\
\hline Slashdot & 0.0049 (3) & $0.0003(4)$ & $0.0000(6)$ & 0.0003 (4) & $0.1455(2)$ & $0.1493(1)$ \\
\hline TMC & $0.4303(3)$ & $0.4335(1)$ & 0.4307 (2) & $0.4175(4)$ & $0.0163(5)$ & $0.0138(6)$ \\
\hline Avg. rank & 3.00 & 3.33 & 4.50 & 4.33 & 2.67 & 3.00 \\
\hline \multicolumn{7}{|c|}{ (c) Hamming score } \\
\hline $20 \mathrm{NG}$ & $0.9523(1)$ & $0.9522(2)$ & $0.9512(3)$ & $0.9511(4)$ & $0.9311(6)$ & $0.9432(5)$ \\
\hline Enron & $0.9416(2)$ & $0.9375(4)$ & $0.9381(3)$ & $0.9419(1)$ & $0.9250(6)$ & $0.9350(5)$ \\
\hline IMDB & $0.9282(4)$ & $0.9284(3)$ & $0.9286(2)$ & $0.9286(1)$ & $0.8886(6)$ & $0.9151(5)$ \\
\hline Ohsumed & $0.9344(1)$ & $0.9341(2)$ & $0.9330(3)$ & $0.9326(4)$ & $0.9224(6)$ & $0.9249(5)$ \\
\hline Slashdot & $0.9461(4)$ & $0.9461(3)$ & $0.9463(1)$ & $0.9463(1)$ & $0.9154(6)$ & $0.9233(5)$ \\
\hline TMC & $0.9154(1)$ & $0.9146(4)$ & $0.9151(2)$ & $0.9149(3)$ & $0.8483(6)$ & $0.8503(5)$ \\
\hline Avg. rank & 2.17 & 3.00 & 2.33 & 2.33 & 6.00 & 5.00 \\
\hline
\end{tabular}

Each table contains the values of the measure (and the rank) of each method on each dataset

\subsection{Results on the example-based measures}

The values and rankings on the example-based measures (accuracy, $\mathrm{F}_{e x}^{1}$ and Hamming score) are presented in Table 2 . The results of the Friedman-Nemenyi significance tests are presented in Fig. 3 in the form of average rank diagrams.

With regards to the comparison of iSOUP model and regression trees, the average rank of model trees is higher than the average rank of regression trees in all example-based measures, even though the difference is not statistically significant. The results on individual datasets in terms of the Hamming score are nearly identical, while model trees are slightly better on the accuracy and $\mathrm{F}_{e x}^{1}$ measures. Even when regression trees beat model trees on a particular dataset, the difference in performance is much smaller than when model trees perform better.

The results of the comparison between the single-tree methods on the example-based evaluation measures are not entirely clear-cut. For both accuracy and $\mathrm{F}_{e x}^{1}$, the average rank of the $H T_{P S}$ method is higher than the average ranks of model and regression trees, but the difference is not statistically significant. The $H T_{P S}$ method has poorer performance on the Enron and TMC datasets, where regression and model trees both outperform $H T_{P S}$. However, the results on the Hamming score show that the average rank of both iSOUP model 


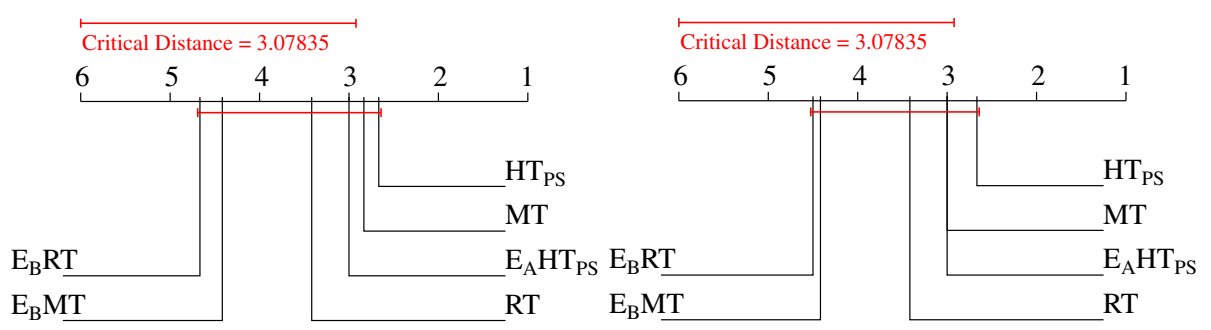

(a)

(b)

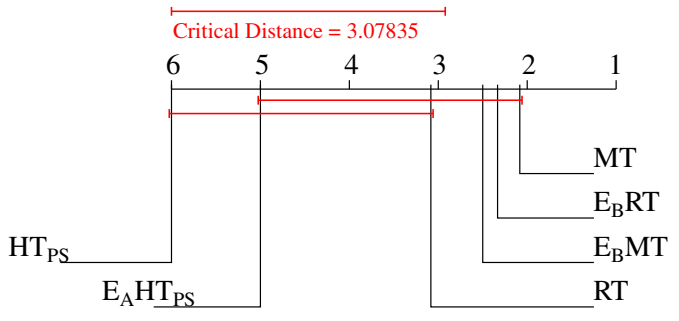

(c)

Fig. 3 Average rank diagrams for the example-based measures. a Accuracy, $\mathbf{b} \mathrm{F}_{e x}^{1}$, c Hamming score

and regression trees are much higher than the rank of the $H T_{P S}$ method. The difference in performance between model trees and the $H T_{P S}$ method is in this case statistically significant.

When examining the performance of the learning methods in terms of the accuracy and $\mathrm{F}_{e x}^{1}$ in detail (per dataset), we again observe very mixed results. It is noticeable that, on some datasets, a group of methods has orders of magnitude better results than the other methods, i.e., $H T_{P S}$ and $E_{A} H T_{P S}$ on the Slashdot dataset, $M T, R T, E_{B M T}$ and $E_{B R T}$ on the Enron dataset, and $E_{A} H T_{P S}$ on the IMDB dataset. We found no statistically significant differences in performance for both the accuracy measure (Fig. 3a) as well as the $\mathrm{F}_{e x}^{1}$ measure (Fig. 3b). On the other hand, the results in terms of the Hamming score are much clearer. $M T, R T$, $E_{B M T}$ and $E_{B R T}$ have higher average rank than $H T_{P S}$ and $E_{A} H T_{P S}$. However, according to the Friedman-Nemenyi post-hoc test, only $H T_{P S}$ is significantly worse than $M T, E_{B R T}$ and $E_{B M T}$ (Fig. 3c).

\subsection{Results on the label-based measures}

The performance measure values and rankings for the label-based measures ( Precision $_{\text {macro }}$, Recall $_{\text {macro }}, \mathrm{F}_{\text {macro }}^{1}$, Precision micro $_{\text {, Recall }}$ micro and $\mathrm{F}_{\text {micro }}^{1}$ ) are presented in Tables 3 and 4. The results of the Friedman-Nemenyi post-hoc significance tests are presented in Fig. 4.

On all macro label-based evaluation measures, model trees get results better than or about equal to the results of regression trees. While regression trees do outperform model trees on some datasets, e.g., for all of the macro measures on the Ohsumed dataset, the differences in these cases are relatively small, while when the model trees outperform regression trees, e.g., for all macro measures on the IMDB dataset, the differences are considerably larger. For all three measures, the difference in average ranks of the methods is not statistically significant.

The results on the micro measures are similar. Model trees have higher average rank than regression trees in terms of Precision $_{\text {micro }}$, though the differences are not statistically significant. The results on Recall micro $_{\text {and }} \mathrm{F}_{\text {micro }}^{1}$ are more scattered, with model still mostly having 
Table 3 Predictive performance results: label-based measures (macro)

\begin{tabular}{|c|c|c|c|c|c|c|}
\hline & $M T$ & $R T$ & $E_{B R T}$ & $E_{B M T}$ & $H T_{P S}$ & $E_{A} H T_{P S}$ \\
\hline \multicolumn{7}{|c|}{ (a) Precision macro measure } \\
\hline $20 N G$ & $0.5527(1)$ & $0.3873(4)$ & $0.3351(5)$ & $0.4279(3)$ & $0.1944(6)$ & $0.4427(2)$ \\
\hline Enron & $0.0679(1)$ & $0.0341(6)$ & $0.0427(5)$ & $0.0588(3)$ & $0.0643(2)$ & $0.0474(4)$ \\
\hline IMDB & $0.2306(2)$ & $0.1452(3)$ & $0.0576(5)$ & $0.2824(1)$ & $0.0392(6)$ & $0.1157(4)$ \\
\hline Ohsumed & $0.2872(2)$ & $0.2946(1)$ & $0.2788(3)$ & $0.2612(4)$ & $0.1653(6)$ & $0.1907(5)$ \\
\hline Slashdot & $0.1347(2)$ & $0.0152(5)$ & $0.0000(6)$ & $0.0227(4)$ & $0.1311(3)$ & $0.1842(1)$ \\
\hline TMC & $0.3321(1)$ & $0.3135(3)$ & $0.3185(2)$ & $0.2380(4)$ & $0.0081(6)$ & $0.0083(5)$ \\
\hline Avg. rank & 1.50 & 3.67 & 4.33 & 3.17 & 4.83 & 3.50 \\
\hline \multicolumn{7}{|c|}{ (b) Recall macro measure } \\
\hline $20 \mathrm{NG}$ & $0.1127(4)$ & $0.1156(3)$ & $0.0667(5)$ & $0.0635(6)$ & $0.3156(1)$ & $0.2787(2)$ \\
\hline Enron & $0.0319(1)$ & $0.0193(4)$ & $0.0205(3)$ & $0.0299(2)$ & $0.0096(5)$ & $0.0019(6)$ \\
\hline IMDB & $0.0060(3)$ & $0.0012(4)$ & $0.0002(6)$ & $0.0010(5)$ & $0.0411(2)$ & 0.0557 (1) \\
\hline Ohsumed & $0.0840(4)$ & $0.0884(3)$ & $0.0495(5)$ & $0.0406(6)$ & $0.1623(1)$ & 0.1433 (2) \\
\hline Slashdot & $0.0021(3)$ & 0.0001 (4) & $0.0000(6)$ & 0.0001 (4) & 0.0861 (1) & $0.0586(2)$ \\
\hline TMC & $0.1237(2)$ & $0.1332(1)$ & $0.1070(3)$ & 0.0981 (4) & $0.0413(5)$ & $0.0388(6)$ \\
\hline Avg. rank & 2.83 & 3.17 & 4.67 & 4.50 & 2.50 & 3.17 \\
\hline \multicolumn{7}{|c|}{ (c) $\mathrm{F}_{\text {macro }}^{1}$ measure } \\
\hline 20NG & $0.1619(4)$ & $0.1630(3)$ & $0.1047(5)$ & $0.0999(6)$ & $0.2287(2)$ & $0.2717(1)$ \\
\hline Enron & $0.0364(1)$ & $0.0199(4)$ & $0.0217(3)$ & $0.0340(2)$ & $0.0127(5)$ & $0.0032(6)$ \\
\hline IMDB & $0.0113(3)$ & 0.0023 (4) & $0.0004(6)$ & 0.0019 (5) & $0.0239(2)$ & $0.0540(1)$ \\
\hline Ohsumed & $0.1210(4)$ & $0.1269(3)$ & $0.0745(5)$ & $0.0617(6)$ & $0.1586(1)$ & $0.1523(2)$ \\
\hline Slashdot & $0.0041(3)$ & $0.0002(5)$ & $0.0000(6)$ & $0.0002(4)$ & $0.0627(1)$ & $0.0487(2)$ \\
\hline TMC & $0.1503(2)$ & $0.1605(1)$ & $0.1228(3)$ & $0.1110(4)$ & $0.0064(5)$ & $0.0041(6)$ \\
\hline Avg. rank & 2.83 & 3.33 & 4.67 & 4.50 & 2.67 & 3.00 \\
\hline
\end{tabular}

Each table contains the values of the measure (and the rank) of each method on each dataset

Table 4 Predictive performance results: label-based measures (micro)

\begin{tabular}{|c|c|c|c|c|c|c|}
\hline & $M T$ & $R T$ & $E_{B R T}$ & $E_{B M T}$ & $H T_{P S}$ & $E_{A} H T_{P S}$ \\
\hline \multicolumn{7}{|c|}{ (a) Precision micro $_{\text {measure }}$} \\
\hline $20 \mathrm{NG}$ & $0.7408(3)$ & $0.7189(4)$ & $0.8227(2)$ & $0.8270(1)$ & $0.3253(6)$ & $0.4218(5)$ \\
\hline Enron & $0.6108(2)$ & $0.5363(4)$ & $0.5539(3)$ & $0.6249(1)$ & $0.0664(6)$ & $0.0863(5)$ \\
\hline IMDB & $0.4411(3)$ & $0.3746(4)$ & $0.5242(2)$ & $0.5864(1)$ & $0.0844(6)$ & $0.3461(5)$ \\
\hline Ohsumed & $0.7561(3)$ & $0.7216(4)$ & $0.8086(2)$ & $0.8189(1)$ & $0.4453(6)$ & $0.4677(5)$ \\
\hline Slashdot & $0.3220(1)$ & $0.0556(5)$ & $0.0000(6)$ & $0.2500(2)$ & $0.1587(4)$ & $0.1923(3)$ \\
\hline TMC & $0.6427(2)$ & $0.6263(4)$ & $0.6394(3)$ & $0.6481(1)$ & $0.0280(5)$ & $0.0248(6)$ \\
\hline Avg. rank & 2.33 & 4.17 & 3.00 & 1.17 & 5.50 & 4.83 \\
\hline \multicolumn{7}{|c|}{ (b) Recall micro $_{\text {measure }}$} \\
\hline $20 \mathrm{NG}$ & $0.1123(4)$ & $0.1151(3)$ & $0.0666(5)$ & $0.0633(6)$ & $0.3161(1)$ & $0.2786(2)$ \\
\hline Enron & $0.2330(1)$ & $0.1424(4)$ & $0.1520(3)$ & $0.2214(2)$ & $0.0136(5)$ & $0.0021(6)$ \\
\hline IMDB & $0.0182(3)$ & $0.0029(4)$ & $0.0006(6)$ & $0.0028(5)$ & $0.0568(2)$ & $0.2123(1)$ \\
\hline
\end{tabular}


Table 4 continued

\begin{tabular}{lllllll}
\hline & $M T$ & $R T$ & $E_{B R T}$ & $E_{B M T}$ & $H T_{P S}$ & $E_{A} H T_{P S}$ \\
\hline Ohsumed & $0.1374(4)$ & $0.1439(3)$ & $0.0965(5)$ & $0.0865(6)$ & $0.2957(1)$ & $0.2762(2)$ \\
Slashdot & $0.0043(3)$ & $0.0002(4)$ & $0.0000(6)$ & $0.0002(4)$ & $0.1341(1)$ & $0.1341(1)$ \\
TMC & $0.3644(2)$ & $0.3803(1)$ & $0.3643(3)$ & $0.3428(4)$ & $0.0149(5)$ & $0.0126(6)$ \\
Avg. rank & 2.83 & 3.17 & 4.67 & 4.50 & 2.50 & 3.00 \\
$\begin{array}{lllll}\text { (c) } \mathrm{F}_{\text {micro }}^{1} \text { measure } \\
\text { 20NG }\end{array}$ & $0.1950(4)$ & $0.1985(3)$ & $0.1232(5)$ & $0.1176(6)$ & $0.3207(2)$ & $0.3356(1)$ \\
Enron & $0.3374(1)$ & $0.2251(4)$ & $0.2385(3)$ & $0.3270(2)$ & $0.0225(5)$ & $0.0041(6)$ \\
IMDB & $0.0350(3)$ & $0.0057(4)$ & $0.0012(6)$ & $0.0056(5)$ & $0.0679(2)$ & $0.2632(1)$ \\
Ohsumed & $0.2325(4)$ & $0.2399(3)$ & $0.1724(5)$ & $0.1564(6)$ & $0.3554(1)$ & $0.3473(2)$ \\
Slashdot & $0.0084(3)$ & $0.0004(5)$ & $0.0000(6)$ & $0.0004(4)$ & $0.1454(2)$ & $0.1580(1)$ \\
TMC & $0.4651(2)$ & $0.4732(1)$ & $0.4642(3)$ & $0.4484(4)$ & $0.0195(5)$ & $0.0167(6)$ \\
Avg. rank & 2.83 & 3.33 & 4.67 & 4.50 & 2.83 & 2.83 \\
\hline
\end{tabular}

Each table contains the values of the measure (and the rank) of each method on each dataset

higher average rank than regression trees. Again, however, the differences in performance when model trees win are considerably larger than when regression trees outperform them.

When comparing the single tree methods, we find that the results on two of the datasets, Enron and TMC, deviate from the rest. Noticeably, on the remaining datasets $H T_{P S}$ outperforms model and regression trees on all measures, with the exception of Precision macro $_{\text {and }}$ Precision $_{\text {micro }}$, while on the Enron and TMC datasets regression and model trees outperform $H T_{P S}$ on all label-based evaluation measures. Additionally, the results for Precision macro $_{\text {and }}$ Precision $_{\text {micro }}$ show that iSOUP single tree methods also outperform $H T_{P S}$ on the remaining datasets.

The comparison of all of the methods in terms of each of the label-based evaluation measures is not straightforward. Ordinary bagging methods (not including $E_{A} H T_{P S}$ ), perform relatively badly according to Recall ${ }_{\text {macro }}$, Recall micro $_{\text {m }}, \mathrm{F}_{\text {macro }}^{1}$ and $\mathrm{F}_{\text {micro }}^{1}$, as can be seen from the average rank diagrams in Fig. 4. While on these measures the differences in rank are not statistically significant, their significance might yield in either direction if experiments are conducted on more datasets. Interestingly, bagging of model trees performs very well in terms of Precision micro, where it statistically significantly outperforms both $H T_{P S}$ and $E_{A} H T_{P S}$. Additionally, model trees also significantly outperform $H T_{P S}$. On the other hand, we only have enough evidence to conclude that $H T_{P S}$ significantly outperforms model trees in terms

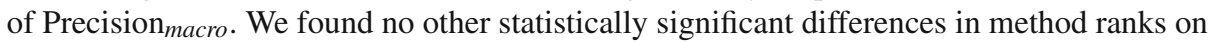
any of the remaining label-based measures.

\subsection{Results on the ranking-based measures}

The performance values and rankings on the ranking-based measures (ranking loss, logarithmic loss and average precision) are presented in Table 5. The results of the Friedman-Nemenyi significance tests are presented in Fig. 5. We note that the calculation of logarithmic loss expects the predicted values to lay in the $[0,1]$ interval and that we have no guarantee that the predictions of model trees will fall on this interval. We further discuss the implications of this fact in the discussion section. 


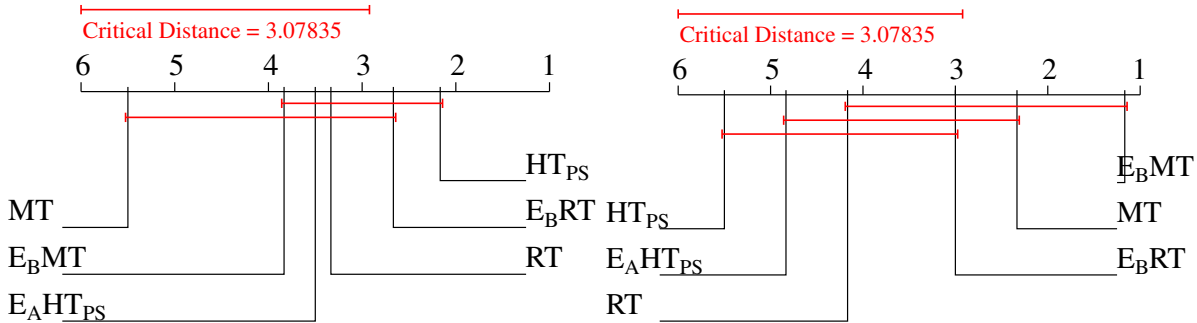

(a)

(b)

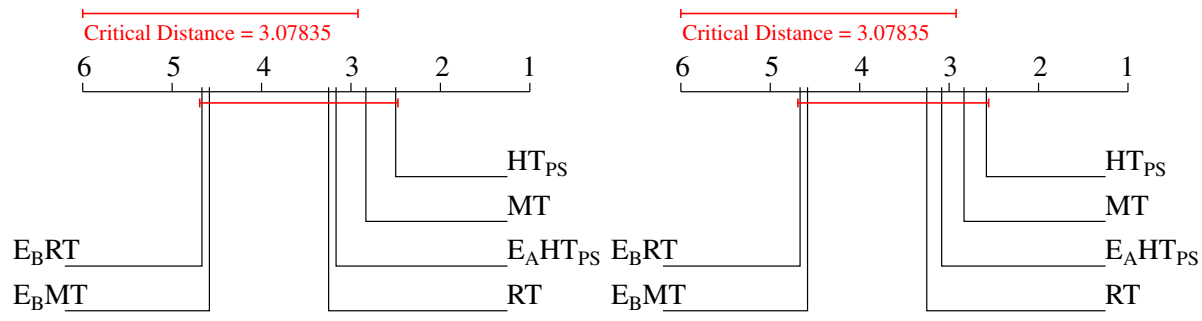

(c)

(d)

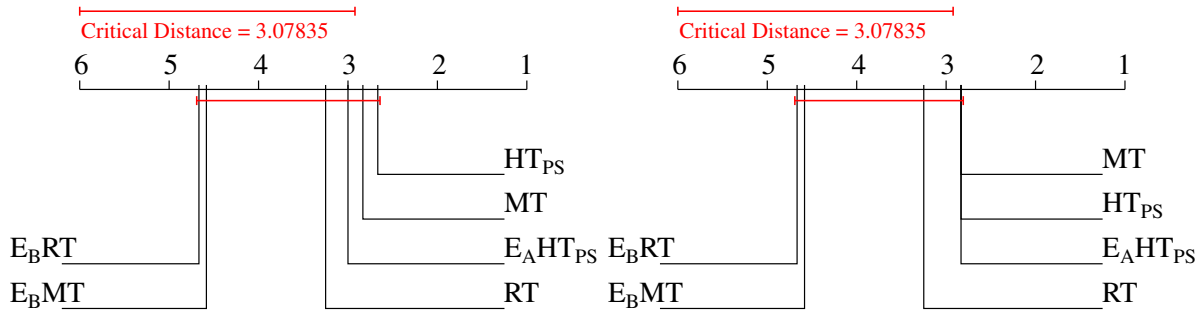

(e)

(f)

Fig. 4 Average ranking diagrams for the label-based measures. a Precision macro, $_{\text {, }}$ brecision micro, $_{\text {, }}$ c Recall macro $_{\text {, }}$ d Recall micro $_{\text {, }} \mathrm{F}_{\text {macro }}^{1}, \mathrm{f} \mathrm{F}_{\text {micro }}^{1}$

The differences between the results of model and regression trees on the ranking-based evaluation measures are very small. There is variation in which type of tree outperforms the other over the different measures. The average rank of regression trees is slightly higher than that of model trees for ranking loss, while the opposite is true for logarithmic loss and average precision. The differences should be further studied by using pairwise statistical tests. Both iSOUP regression and model trees outperform $H T_{P S}$ in terms of ranking loss and logarithmic loss (and the difference in performance is statistically significant). In terms of average precision, their results are very close with each of the methods performing best on some of the datasets.

Finally, the ranking diagram for the algorithms in terms of ranking loss shows that bagging with model trees generally performs best on all of the datasets, followed by bagging of regression trees, regression and model trees, and finally $E_{A} H T_{P S}$ and $H T_{P S}$. In terms of statistical significance, bagging of model trees is better than $H T_{P S}$ and $E_{A} H T_{P S}$, and bagging of regression trees is better than $H T_{P S}$ (Fig. 5a). The results in terms of logarithmic loss are 
Table 5 Predictive performance results: ranking-based measures

\begin{tabular}{|c|c|c|c|c|c|c|}
\hline & $M T$ & $R T$ & $E_{B R T}$ & $E_{B M T}$ & $H T_{P S}$ & $E_{A} H T_{P S}$ \\
\hline \multicolumn{7}{|c|}{ (a) Ranking loss } \\
\hline $20 \mathrm{NG}$ & $0.2672(4)$ & $0.2768(5)$ & $0.2272(2)$ & $0.2271(1)$ & $0.3852(6)$ & $0.2545(3)$ \\
\hline Enron & $0.1208(4)$ & $0.1181(2)$ & $0.1183(3)$ & $0.1165(1)$ & $0.3474(6)$ & $0.3430(5)$ \\
\hline IMDB & 0.1878 (4) & $0.1737(3)$ & 0.1708 (2) & $0.1705(1)$ & $0.5912(6)$ & $0.2615(5)$ \\
\hline Ohsumed & $0.2254(4)$ & $0.2163(3)$ & 0.2024 (1) & $0.2110(2)$ & $0.3752(6)$ & $0.3102(5)$ \\
\hline Slashdot & $0.2202(2)$ & $0.2216(4)$ & $0.2206(3)$ & $0.2185(1)$ & $0.4801(6)$ & $0.3760(5)$ \\
\hline $\mathrm{TMC}$ & $0.1220(4)$ & $0.1158(3)$ & $0.1012(1)$ & $0.1132(2)$ & $0.4688(5)$ & $0.4820(6)$ \\
\hline Avg. rank & 3.67 & 3.33 & 2.00 & 1.33 & 5.83 & 4.83 \\
\hline \multicolumn{7}{|c|}{ (b) Logarithmic loss } \\
\hline $20 \mathrm{NG}$ & $0.1771(3)$ & $0.1785(4)$ & 0.1648 (1) & $0.1671(2)$ & $0.6799(6)$ & $0.2923(5)$ \\
\hline Enron & 0.1565 (1) & $0.1697(4)$ & $0.1693(3)$ & $0.1574(2)$ & $0.5582(6)$ & $0.4736(5)$ \\
\hline IMDB & $0.2089(1)$ & $0.2147(4)$ & $0.2104(3)$ & $0.2101(2)$ & $1.3033(6)$ & $0.4726(5)$ \\
\hline Ohsumed & $0.2293(4)$ & $0.2279(3)$ & $0.2103(1)$ & $0.2148(2)$ & $0.7401(6)$ & $0.4860(5)$ \\
\hline Slashdot & $0.1824(1)$ & 0.1857 (4) & $0.1839(3)$ & $0.1834(2)$ & $0.6972(6)$ & $0.4575(5)$ \\
\hline TMC & $0.2328(4)$ & $0.2290(3)$ & $0.2128(1)$ & $0.2212(2)$ & $1.5564(6)$ & $1.3226(5)$ \\
\hline Avg. rank & 2.33 & 3.67 & 2.00 & 2.00 & 6.00 & 5.00 \\
\hline \multicolumn{7}{|c|}{ (c) Average precision } \\
\hline $20 \mathrm{NG}$ & $0.1793(5)$ & $0.1755(6)$ & $0.1900(2)$ & $0.1928(1)$ & $0.1831(3)$ & $0.1816(4)$ \\
\hline Enron & $0.1131(1)$ & $0.1023(4)$ & $0.1024(3)$ & $0.1125(2)$ & $0.0739(6)$ & $0.0750(5)$ \\
\hline IMDB & $0.1986(2)$ & $0.1901(5)$ & 0.1907 (4) & $0.1972(3)$ & $0.2385(1)$ & $0.1758(6)$ \\
\hline Ohsumed & $0.1846(2)$ & $0.1806(4)$ & $0.1836(3)$ & 0.1848 (1) & $0.1674(6)$ & $0.1748(5)$ \\
\hline Slashdot & $0.1586(3)$ & $0.1529(6)$ & 0.1585 (4) & $0.1565(5)$ & $0.1871(2)$ & $0.1951(1)$ \\
\hline TMC & 0.1992 (4) & $0.2001(3)$ & $0.2121(1)$ & $0.2016(2)$ & $0.1698(6)$ & 0.1708 \\
\hline Avg. rank & 2.83 & 4.67 & 2.83 & 2.33 & 4.00 & 4.33 \\
\hline
\end{tabular}

Each table contains the values of the measure (and the rank) of each method on each dataset

very similar. Here, bagging of model trees, bagging of regression trees, as well as single model trees, statistically significantly outperform $H T_{P S}$ (Fig. 5b). The results for average precision are mixed, with different methods taking first and last rank on different datasets (Fig. 5c). Hence, no statistically significant differences were observed.

\subsection{Results on the efficiency measures}

The values and rankings on the efficiency measures (memory and time use) are presented in Table 6. The results of the Friedman-Nemenyi significance tests are presented in Fig. 6.

The expected result of model trees using both more memory and time is evident from Table 6. While the difference in memory use is relatively small, the time use is increased by about $10-20 \%$ when using model trees.

$H T_{P S}$ uses considerably less memory when compared to model and regression trees. In terms of time use, it performs better than iSOUP single trees on some datasets, while it uses considerably more time on others. The differences in time are most likely due to the pruned sets procedure, as the base tree learning steps are similar between the methods. 


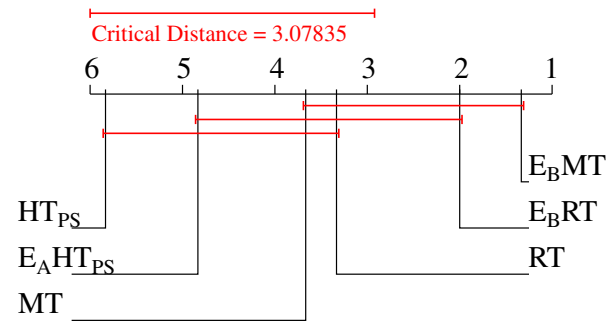

(a)

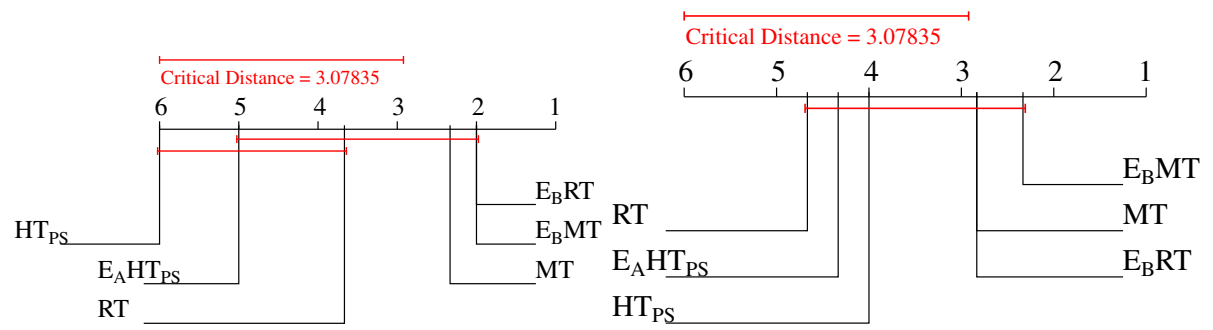

(b)

(c)

Fig. 5 Average rank diagrams for the ranking-based measures. a Ranking loss, b logarithmic loss, $\mathbf{c}$ average precision

Overall, regression trees appear to be the quickest method, while $H T_{P S}$ appears to be the least memory intensive. We observe several statistically significant differences and (eventhough some can be inferred by common sense) report all of them. Specifically, $H T_{P S}$ uses less memory than bagging of regression trees and bagging of model trees, regression trees and $E_{A} H T_{P S}$ use less memory than bagging of model trees, regression trees are quicker than bagging of model trees and $E_{A} H T_{P S}$, and both $M T$ and $H T_{P S}$ are quicker than $E_{A} H T_{P S}$ (Fig. 6).

\subsection{Discussion}

We start with a few general remarks, then proceed to address each of the experimental questions in turn.

First, recall that both example-based and label-based measures depend on the selected threshold. While threshold selection is far from a trivial task in the multi-label classification scenario and its difficulty is further compounded in the setting of the data stream mining, it is expected that a better selection of threshold would increase the performance of any classifier. Whether the selection is best done on a method-by-method basis, dataset-by-dataset basis or even for each method-dataset pair requires substantial further investigation. Due to this, we place a stronger emphasis on the threshold-independent ranking-based evaluation measures.

We also observe that logarithmic loss is perhaps not the best measure to evaluate model trees and their ensembles in the MLC via MTR setting. The fact that a model tree can predict a value higher than 1 or lower than 0 , means that it can be rewarded for predictions for which it is "very sure", if we look at the predicted value as a probability. However, this cuts both ways, as a prediction of a negative value for a label that is present will also result in a 
Table 6 Efficiency results: memory and time usage

\begin{tabular}{|c|c|c|c|c|c|c|}
\hline & $M T$ & $R T$ & $E_{B R T}$ & $E_{B M T}$ & $H T_{P S}$ & $E_{A} H T_{P S}$ \\
\hline \multicolumn{7}{|c|}{ (a) Memory (MB) } \\
\hline $20 \mathrm{NG}$ & $101.61(4)$ & $101.44(3)$ & $970.35(5)$ & $972.03(6)$ & $3.43(1)$ & $22.46(2)$ \\
\hline Enron & $10.20(3)$ & $9.77(2)$ & $99.92(5)$ & $104.20(6)$ & $6.56(1)$ & $56.82(4)$ \\
\hline IMDB & $382.33(4)$ & 382.08 (3) & $3575.54(5)$ & $3578.03(6)$ & $22.53(1)$ & $39.31(2)$ \\
\hline Ohsumed & $191.29(4)$ & 191.09 (3) & $1368.17(5)$ & $1370.13(6)$ & $3.63(1)$ & $32.89(2)$ \\
\hline Slashdot & $15.45(3)$ & $15.26(2)$ & $140.02(5)$ & $141.94(6)$ & $3.72(1)$ & $26.03(4)$ \\
\hline $\mathrm{TMC}$ & $36.75(4)$ & $36.65(3)$ & 304.34 (5) & $305.31(6)$ & $2.30(1)$ & $18.62(2)$ \\
\hline Avg. rank & 3.67 & 2.67 & 5.00 & 6.00 & 1.00 & 2.67 \\
\hline \multicolumn{7}{|l|}{ (b) Time (s) } \\
\hline $20 \mathrm{NG}$ & $32.95(2)$ & $28.59(1)$ & 196.17 (4) & $230.09(5)$ & $51.90(3)$ & $723.22(6)$ \\
\hline Enron & $7.71(3)$ & $6.56(2)$ & $40.97(5)$ & $48.62(6)$ & $1.93(1)$ & $13.11(4)$ \\
\hline IMDB & $277.06(2)$ & $250.86(1)$ & $2434.20(4)$ & $2971.10(5)$ & $344.45(3)$ & $4723.53(6)$ \\
\hline Ohsumed & $27.85(2)$ & $25.31(1)$ & $168.74(4)$ & $195.32(5)$ & $39.73(3)$ & $632.72(6)$ \\
\hline Slashdot & $7.13(2)$ & $5.82(1)$ & $41.51(4)$ & $48.59(5)$ & $9.03(3)$ & $78.27(6)$ \\
\hline TMC & $26.13(3)$ & $22.35(2)$ & $150.52(4)$ & $188.41(5)$ & $14.49(1)$ & $259.11(6)$ \\
\hline Avg. rank & 2.33 & 1.33 & 4.17 & 5.17 & 2.33 & 5.67 \\
\hline
\end{tabular}

Each table contains the values of the efficiency measure (and the rank) of each method on each dataset

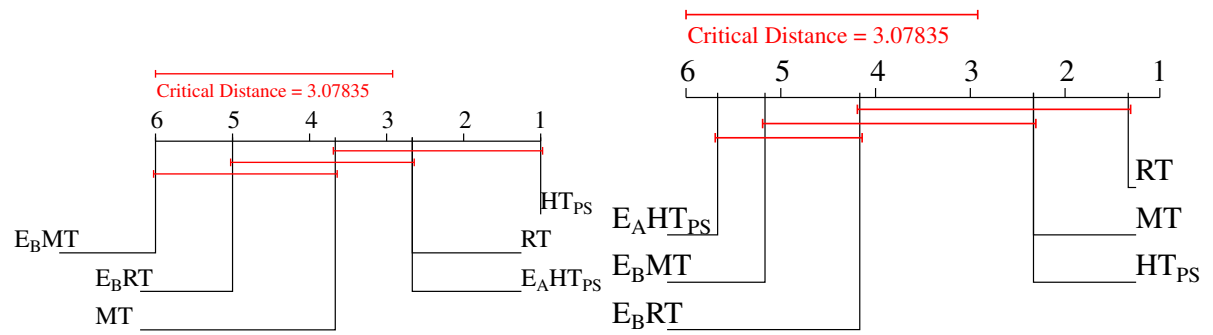

(a)

(b)

Fig. 6 Average rank diagrams for the efficiency measures. a Memory consumption, b time consumption

more severe penalty in terms of the logarithmic loss. Due to the learning procedure, we do, however, expect that the former will occur more often than the latter.

Regarding the first experimental question, whether model trees with the adaptive perceptron outperform regression trees, we can conclude based on the above results that some improvement can be gained by using model trees. However, we also note that using model trees increases the use of resources, which can be a limiting factor when choosing a method for a real-life application. While the increase in memory use is relatively small, model tree construction consumes about 10-20\% more time as compared to regression trees.

Continuing to the second question of how iSOUP single model and regression trees compare to Hoeffding trees with pruned sets, we observe that, on the example-based and label-based evaluation measures, all of the trees have similar performance. The only exception is the Hamming score. However, based on the observations made on the ranking-based methods, we conclude that both model and regression iSOUP trees either perform as well as 
or outperform the Hoeffding trees with pruned sets, though these findings should be further confirmed by a rigorous statistical testing.

Looking at the results of the comparisons of both single tree and ensemble methods along the various evaluation measures, we find that different methods achieve the best results for different evaluation measures. This is to be expected, as the different learning procedures inevitably optimize different quantities. This in turn influences the performance evaluation for a given evaluation measure, depending on the similarity of the optimized quantity and the evaluation measure.

We did find some statistically significant differences, most notably for the ranking-based ranking loss and logarithmic loss measures. The bagging ensemble of iSOUP model trees outperformed both single tree and ensembles of Hoeffding trees with pruned sets for the first measure and only single Hoeffding trees with pruned sets for the second. These significant differences are very important, as they ranking-based measures in question are threshold independent, while measures like precision and recall can be traded off by setting different thresholds.

Finally, we make conclusions with regard to resource consumption. The results show that, in general, the additional use of resources by the more complex methods, like model trees or ensembles of models, does contribute to better performance. This justifies the use of more complex models. However, we must be aware that there are associated costs that need to be considered. This is especially relevant for real-world applications, where such methods might be made to operate on low-memory or computationally slow devices. To this end, we can recommend the use of memory conservation techniques, such as those proposed by Ikonomovska et al. (2010).

\section{Conclusion and future work}

In this paper, we have introduced the multi-label classification via multi-target regression methodology for learning from data streams. We have also introduced the iSOUP-Tree algorithm that utilizes this methodology to address the multi-label classification task by using multi-target regression and model trees. We have performed experiments on several multilabel datasets, to address a number of experimental questions concerning the proposed method and its competitors.

First, we have shown that iSOUP model trees is perform better than iSOUP regression trees for a large set of evaluation measures for multi-label classification. For two measures they perform worse. In both cases, the difference is not statistically significant. Th better performance of model trees might be due to the use of the adaptive perceptron. In contrast, in our earlier work the use of regular perceptrons decreased the performance of model trees as compared to the performance of regression trees (Osojnik et al. 2015).

Next, we compared our method in a single tree (non-ensemble) setup to Hoeffding trees with pruned sets (Read et al. 2012). The thresholding aspect of MLC made clear conclusions for some of the observed measures elusive. Still, we can observe that the performance of iSOUP model and regression trees is better than that of Hoeffding trees with pruned sets with respect to two ranking-based measures (ranking loss and logarithmic loss), even though the difference in performance is not statistically significant.

We continued with a wider comparison with some additional methods. Specifically, we included bagging of iSOUP model trees and regression trees, as well as ADWIN bagging of Hoeffding trees with pruned sets. Bagging of iSOUP model trees method performed the best in terms of threshold-independent ranking-based evaluation measures (ranking loss, 
logarithmic loss and average precision), with some of the differences in performance being significant. However, further experiments on a larger collection of datasets would be needed to yield stronger statistical confirmation of our findings.

For our experimental comparison, we considered a variety of evaluation measures commonly used in the MLC setting. We obtained the clearest conclusions when using ranking-based measures, which we consider most relevant for MLC, in addition to being threshold-free. However, in real-world applications, any of the considered measures could be a criterion which determines success. The measures we considered are widely used in the MLC community, and showcase the strengths and weaknesses of the observed algorithms.

We have shown that the use of more complex methods yields better predictive performance. However, this comes at the cost of greater use of resources. This should be considered when designing real-world applications, where resources may be limited and a simpler method might be more suitable.

We encountered several interesting avenues of further work. The inescapable problem in multi-label classification is the problem of thresholding. While thresholding is a key piece of the MLC via MTR methodology, it is also present in many methods which directly address the MLC task. Exploring whether a single threshold is appropriate for all of the labels, or whether multiple thresholds, one per label, should be used, is a promising line of future work. Specifically, examining the thresholding strategies of Tsoumakas and Katakis (2007) and Largeron et al. (2012) as well as the work of Triguero and Vens (2016) and determining if and how their results can be applied in the streaming setting will be our first step along this avenue.

Additionally, we wish to address the task of change detection and adaptation in the iSOUPTree method directly, without relying on the use of ensemble methods such as ADWIN bagging. As we have shown, the use of ensembles can incur considerably higher use of resources, which might not be suitable for all applications. We plan to explore both mechanisms that deal with targets/labels on a one by one basis, as well as those that consider all of the targets together. The adaptation of approaches for change detection and adaptation from the single-target scenario to the multi-target scenario, e.g., from the work of Ikonomovska et al. (2011b), would be a good first step.

Finally, we plan to extend our MLC via MTR methodology and the iSOUP-Tree method to also address the task of hierarchical multi-label classification (HMC), which is increasingly common in the batch learning scenario. In HMC, the labels are ordered in a hierarchy and adhere to the hierarchy constraint, i.e., if an example is labeled with a label it also has to be labelled with the label's ancestors. One way to achieve this would be to extend iSOUP-Tree to deal with the task of hierarchical multi-target regression.

Acknowledgements We would like to acknowledge the support of the EC through the projects: MAESTRA (FP7-ICT-612944) and HBP (FP7-ICT-604102), and the Slovenian Research Agency through a young researcher Grant and the program Knowledge Technologies (P2-0103).

Open Access This article is distributed under the terms of the Creative Commons Attribution 4.0 International License (http://creativecommons.org/licenses/by/4.0/), which permits unrestricted use, distribution, and reproduction in any medium, provided you give appropriate credit to the original author(s) and the source, provide a link to the Creative Commons license, and indicate if changes were made.

\section{Appendix: Evaluation measures for multi-label classification}

In the following definitions, $N$ is the number of examples in the evaluation sample, $\mathcal{L}$ is the set of all labels and $Q=|\mathcal{L}|$ is the number of labels in the provided MLC setting. $\hat{y}_{i}$ and 
$y_{i}$ always stand for the predicted scores and actual labelset of the $i$ th example, respectively, though the latter is used interchangeably with it's indicator vector, i.e., a vector representation of the labelset, where the present labels have a value of 1 while the rest have a value of $0 . \hat{y}$ and $y$ refer to a prediction made by a method on some specific (non-indexed) example and its actual labelset, respectively, and are used to define loss functions. $\hat{y}_{i}^{j}$ refers to the predicted score for the $j$ th label, while $\hat{y}_{i}^{\lambda}$ similarly refers to the predicted score for the label $\lambda$.

For all example-based and label-based measures, $\hat{y}_{i}$ is assumed to already be thresholded, i.e.,

$$
\hat{y}_{i}^{j}= \begin{cases}1 ; & \text { if } \hat{y}_{i}^{j} \geq \tau \text { holds for the predicted score } \hat{y}_{i}^{j}, \\ 0 ; & \text { otherwise }\end{cases}
$$

while the ranking-based measures are computed without thresholding.

\section{Example based measures}

Accuracy The accuracy for an example with a prediction $\hat{y}$ and a real labelset $y$ is defined as the Jaccard similarity coefficient between them, i.e., $\frac{|\hat{y} \cap y|}{|\hat{y} \cup y|}$. The accuracy over a sample is the averaged accuracy over all examples:

$$
\text { Accuracy }=\frac{1}{N} \sum_{i=1}^{N} \frac{\left|\hat{y}_{i} \cap y_{i}\right|}{\left|\hat{y}_{i} \cup y_{i}\right|} .
$$

The higher the accuracy of a model the better its predictive performance.

$\mathrm{F}^{1}$ measure. The $\mathrm{F}^{1}$ measure for MLC is the natural extension of $\mathrm{F}^{1} \mathrm{used}$ in regular classification, however, we can approach it from either the example-based or label-based perspective. The general formula for calculating $\mathrm{F}^{1}$ is the usual harmonic mean of the precision and recall

$$
\mathrm{F}^{1}=\frac{2 \text { Precision } \cdot \text { Recall }}{\text { Precision }+ \text { Recall }}
$$

If we want to calculate $\mathrm{F}^{1}$ from the example-based perspective, we define precision and recall as follows:

$$
\operatorname{Precision}_{e x}=\frac{1}{N} \sum_{i=1}^{N} \frac{\left|\hat{y}_{i} \cap y_{i}\right|}{\left|y_{i}\right|} \operatorname{Recall}_{e x}=\frac{1}{N} \sum_{i=1}^{N} \frac{\left|\hat{y}_{i} \cap y_{i}\right|}{\left|\hat{y}_{i}\right|},
$$

resulting in the final definition for example-based $\mathrm{F}^{1}$ measure

$$
\mathrm{F}_{e x}^{1}=\frac{2 \text { Precision }_{e x} \cdot \text { Recall }_{e x}}{\text { Precision }_{e x}+\operatorname{Recall}_{e x}} .
$$

The definitions of the label-based $\mathrm{F}^{1}$ measures are found in the following section.

Hamming loss The Hamming loss measures how many times an example-label pair is misclassified. Specifically, each label that is either predicted but not real, or vice versa, carries a penalty to the score. The Hamming loss of a single example is the number of such misclassified labels divided by the number of all labels, i.e., $\frac{1}{Q}|\hat{y} \triangle y|$ where $\hat{y} \Delta y$ is the symmetric difference of the sets $\hat{y}$ and $y$. The Hamming loss of a sample is the averaged Hamming loss over all examples:

$$
\text { HammingLoss }=\frac{1}{N} \sum_{i=1}^{N} \frac{1}{Q}\left|\hat{y}_{i} \triangle y_{i}\right|
$$


The Hamming loss of a perfect model, which makes completely correct predictions, is 0 and the lower the Hamming loss the better the predictive performance of a model. Note that the Hamming loss will generally be reported as the Hamming score, i.e., HammingScore = 1 - HammingLoss.

\section{Label-based measures}

To define many of the label-based measures, we expand the definitions of several quantities from regular classification, i.e., the true positive (TP), false positive (FP), true negative (TN) and false negative $(\mathrm{FN})$ rates are each defined on a per-label basis as follows:

$$
\begin{aligned}
T P_{j} & =\left|\left\{\hat{y}_{i} \mid l_{j} \in y_{i} \wedge l_{j} \in \hat{y}_{i}, 1 \leq i \leq N\right\}\right| \\
F P_{j} & =\left|\left\{\hat{y}_{i} \mid l_{j} \notin y_{i} \wedge l_{j} \in \hat{y}_{i}, 1 \leq i \leq N\right\}\right| \\
T N_{j} & =\left|\left\{\hat{y}_{i} \mid l_{j} \notin y_{i} \wedge l_{j} \notin \hat{y}_{i}, 1 \leq i \leq N\right\}\right| \\
F N_{j} & =\left|\left\{\hat{y}_{i} \mid l_{j} \in y_{i} \wedge l_{j} \notin \hat{y}_{i}, 1 \leq i \leq N\right\}\right|,
\end{aligned}
$$

where $1 \leq j \leq Q$ indexes the labels. This further allows us to extend the definitions of precision, recall and $\mathrm{F}^{1}$ in a label-based manner. However, we have two choices how to combine the contributions of each label, macro- and micro-averaging. In macro-averaging, we compute each measure per label and then average the measures over all of the labels, while in micro-averaging, we first sum up TP, FP, TN and FN values and use those to calculate the measures.

Macro-averaged measures The macro-averaged measures are defined as follows:

$$
\begin{aligned}
\text { Precision }_{\text {macro }} & =\frac{1}{Q} \sum_{j=1}^{Q} \frac{T P_{j}}{T P_{j}+F P_{j}} \\
\text { Recall }_{\text {macro }} & =\frac{1}{Q} \sum_{j=1}^{Q} \frac{T P_{j}}{T P_{j}+F N_{j}}
\end{aligned}
$$

To define the macro-averaged $\mathrm{F}^{1}$ measure, we further extend the definition by substituting the precision and recall formulas with their forms in terms of $T P_{j}, F P_{j}, T N_{j}$ and $F N_{j}$, resulting in the formula

$$
\mathrm{F}_{\text {macro }}^{1}=\frac{1}{Q} \sum_{j=1}^{Q} \frac{2 \frac{T P_{j}}{T P_{j}+F P_{j}} \frac{T P_{j}}{T P_{j}+F N_{j}}}{\frac{T P_{j}}{T P_{j}+F P_{j}}+\frac{T P_{j}}{T P_{j}+F N_{j}}}=\frac{1}{Q} \sum_{j=1}^{Q} \frac{2}{2 T P_{j}+F P_{j}+F N_{j}}
$$

Micro-averaged measures The following micro-averaged equations are all obtained using the procedure outlined above:

$$
\begin{aligned}
\text { Precision }_{\text {micro }} & =\frac{\sum_{j=1}^{Q} T P_{j}}{\sum_{j=1}^{Q} T P_{j}+\sum_{j=1}^{Q} F P_{j}} \\
\text { Recall }_{\text {micro }} & =\frac{\sum_{j=1}^{Q} T P_{j}}{\sum_{j=1}^{Q} T P_{j}+\sum_{j=1}^{Q} F N_{j}} \\
\mathrm{~F}_{\text {micro }}^{1} & =\frac{2 \text { Precision }_{\text {micro }} \cdot \text { Recall }_{\text {micro }}}{\text { Precision }_{\text {micro }}+\text { Recall }_{\text {micro }}}
\end{aligned}
$$




\section{Ranking-based measures}

Since thresholding has a significant impact on performance measures and determining the optimal threshold is non-trivial, we use measures that are independent of the chosen threshold. These include ranking loss, logarithmic loss and average precision.

Ranking loss The ranking loss (RankLoss) measure is defined as

$$
\text { RankLoss }=\frac{1}{N} \sum_{i=1}^{N} \frac{\left|D_{i}\right|}{\left|y_{i}\right|\left|\bar{y}_{i}\right|}
$$

where $\bar{y}_{i}=\mathcal{L} \backslash y_{i}$ is the complement of $y_{i}$ in $\mathcal{L}, D_{i}=\left\{\left(\lambda_{k}, \lambda_{l}\right) \mid \hat{y}_{i}^{k} \leq \hat{y}_{i}^{l},\left(\lambda_{k}, \lambda_{l}\right) \in y_{i} \times \bar{y}_{i}\right\}$. Note that these values are now considered without applying thresholding. Essentially, it measures how well the labels are ordered by score, i.e., the loss is low when the labels that aren't present have lower scores than the present labels. Consequently, lower values of ranking loss indicate better performance.

Logarithmic loss Another ranking-based measure is the logarithmic loss (LogLoss) (Read et al. 2011). When calculating LogLoss, each labelling error is graded according to the confidence of the prediction, i.e., low confidence errors result in logarithmically smaller penalties than high confidence errors. Specifically, LogLoss is calculated as

$$
\text { LogLoss }=\frac{1}{N Q} \sum_{i=1}^{N} \sum_{j=1}^{Q} \min \left(-\log -\operatorname{loss}\left(\hat{y}_{i}^{j}, y_{i}^{j}\right), \log N\right),
$$

where $\log \operatorname{loss}(\hat{y}, y)$ is defined as

$$
\log \operatorname{loss}(\hat{y}, y)=y \log \hat{y}+(1-y) \log (1-\hat{y}) .
$$

Here, $\hat{y}_{i}^{j}$ are assumed to be on the $[0,1]$ interval and can essentially be interpreted as posterior probabilities of each label $j$. To prevent a small poorly predicted labelset from greatly distorting the overall error, the minimum of the calculated $\log$ - $\operatorname{loss}$ and $\log N$ is taken. This in essence caps the maximal penalty any label-example pair can contribute. The LogLoss of a perfect classifier is 0 and lower values are desired.

Average precision Let us define $\operatorname{rank}(\hat{y}, \lambda)$ as the ranking of the label $\lambda$ according to the prediction $\hat{y}$, i.e.,

$$
\operatorname{rank}(\hat{y}, \lambda)=1+\left|\left\{\lambda^{\prime} \mid y^{\lambda^{\prime}}>y^{\lambda}, \lambda^{\prime} \in \mathcal{L}\right\}\right| .
$$

This means that if a given label $\lambda$ has the highest predicted value in $\hat{y}_{i}$ of all the labels, it's rank will be 1 , as no other label has a higher predicted value. The average precision (AvgPrecision) measure considers the average fraction of labels ranked above an actually present label $\lambda \in y_{i}$ in a given example. More specifically, average precision is defined as follows:

$$
\text { AvgPrecision }=\frac{1}{N} \sum_{i=1}^{N} \frac{1}{\left|y_{i}\right|} \sum_{\lambda \in y_{i}} \frac{\left|\mathcal{L}_{i}^{\lambda}\right|}{\operatorname{rank}\left(x_{i}, \lambda\right)},
$$

where $\mathcal{L}_{i}^{\lambda}=\left\{l^{\prime} \mid \operatorname{rank}\left(\hat{y}_{i}, \lambda^{\prime}\right) \leq \operatorname{rank}\left(\hat{y}_{i}, \lambda\right), \lambda^{\prime} \in \mathcal{L}\right\}$, i.e., the set of all labels ranked lower than $\lambda$ in $\hat{y}_{i}$. The perfect average precision value is 1 and higher values are desired. 


\section{References}

Appice, A., \& Džeroski, S. (2007). Stepwise induction of multi-target model trees. In Machine Learning: ECML 2007, LNCS (Vol. 4701, pp. 502-509). Springer.

Bifet, A., \& Gavaldà, R. (2009). Adaptive learning from evolving data streams. In Advances in Intelligent Data Analysis VIII (pp. 249-260). Springer.

Bifet, A., Holmes, G., Kirkby, R., \& Pfahringer, B. (2010). Moa: Massive online analysis. The Journal of Machine Learning Research, 11, 1601-1604.

Bifet, A., Holmes, G., Pfahringer, B., Kirkby, R., \& Gavaldà, R. (2009). New ensemble methods for evolving data streams. In Proceedings of the 15th ACM SIGKDD international conference on knowledge discovery and data mining (pp. 139-148). ACM.

Cheng, W., \& Hüllermeier, E. (2009). Combining instance-based learning and logistic regression for multilabel classification. Machine Learning, 76(2-3), 211-225.

Demšar, J. (2006). Statistical comparisons of classifiers over multiple data sets. The Journal of Machine Learning Research, 7, 1-30.

Domingos, P., \& Hulten, G. (2000). Mining high-speed data streams. In Proceedings of the sixth ACM SIGKDD international conference on Knowledge discovery and data mining (pp. 71-80). ACM.

Duarte, J., \& Gama, J. (2014). Ensembles of adaptive model rules from high-speed data streams. In $3 \mathrm{rd}$ international workshop on big data, streams and heterogeneous source mining (pp. 198-213).

Duarte, J., \& Gama, J. (2015). Multi-target regression from high-speed data streams with adaptive model rules. In IEEE international conference on data science and advanced analytics (DSAA), 2015. 366782015 (pp. 1-10).

Frank, E., Wang, Y., Inglis, S., Holmes, G., \& Witten, I. H. (1998). Using model trees for classification. Machine Learning, 32(1), 63-76.

Friedman, M. (1940). A comparison of alternative tests of significance for the problem of $m$ rankings. The Annals of Mathematical Statistics, 11(1), 86-92.

Fürnkranz, J., Hüllermeier, E., Mencía, E. L., \& Brinker, K. (2008). Multilabel classification via calibrated label ranking. Machine Learning, 73(2), 133-153.

Gama, J. (2010). Knowledge discovery from data streams. Boca Raton: CRC Press.

Gibaja, E., \& Ventura, S. (2015). A tutorial on multilabel learning. ACM Computing Surveys (CSUR), 47(3), 52.

Gonçalves, E. C., Plastino, A., \& Freitas, A. A. (2013). A genetic algorithm for optimizing the label ordering in multi-label classifier chains. In 2013 IEEE 25th international conference on tools with artificial intelligence (ICTAI) (pp. 469-476). IEEE.

Hulten, G., \& Domingos, P. (2003). VFML-A toolkit for mining high-speed time-changing data streams. http://www.cs.washington.edu/dm/vfml/.

Ikonomovska, E., Gama, J., \& Džeroski, S. (2010). Learning model trees from evolving data streams. Data Mining and Knowledge Discovery, 23(1), 128-168.

Ikonomovska, E., Gama, J., \& Džeroski, S. (2011a). Incremental multi-target model trees for data streams. In Proceedings of the 2011 ACM symposium on applied computing (pp. 988-993). ACM.

Ikonomovska, E., Gama, J., \& Džeroski, S. (2011b). Learning model trees from evolving data streams. Data Mining and Knowledge Discovery, 23(1), 128-168.

Ikonomovska, E., Gama, J., \& Džeroski, S. (2015). Online tree-based ensembles and option trees for regression on evolving data streams. Neurocomputing, 150, 458-470.

Largeron, C., Moulin, C., \& Géry, M. (2012). MCut: A thresholding strategy for multi-label classification (pp. 172-183). Berlin: Springer.

Madjarov, G., Kocev, D., Gjorgjevikj, D., \& Džeroski, S. (2012). An extensive experimental comparison of methods for multi-label learning. Pattern Recognition, 45(9), 3084-3104.

Nemenyi, P. (1963). Distribution-free multiple comparisons. PhD thesis, New Jersey.

Osojnik, A., Panov, P., \& Džeroski, S. (2015). Multi-label classification via multi-target regression on data streams. In Discovery Science (pp. 170-185). Springer.

Oza, N. C. (2005). Online bagging and boosting. In 2005 IEEE international conference on systems, man and cybernetics (Vol. 3, pp. 2340-2345). IEEE.

Qu, W., Zhang, Y., Zhu, J., \& Qiu, Q. (2009). Mining multi-label concept-drifting data streams using dynamic classifier ensemble. In Advances in machine learning (pp. 308-321). Springer.

Read, J. (2008). A pruned problem transformation method for multi-label classification. In Proceedings of 2008 New Zealand computer science research student conference (NZCSRS 2008) (pp. 143-150).

Read, J., Bifet, A., Holmes, G., \& Pfahringer, B. (2012). Scalable and efficient multi-label classification for evolving data streams. Machine Learning, 88(1-2), 243-272. 
Read, J., Pfahringer, B., \& Holmes, G. (2008). Multi-label classification using ensembles of pruned sets. In Eighth IEEE international conference on data mining, 2008. ICDM'08 (pp. 995-1000). IEEE.

Read, J., Pfahringer, B., Holmes, G., \& Frank, E. (2009). Classifier chains for multi-label classification. Berlin: Springer.

Read, J., Pfahringer, B., Holmes, G., \& Frank, E. (2011). Classifier chains for multi-label classification. Machine learning, 85(3), 333-359.

Rutkowski, L., Pietruczuk, L., Duda, P., \& Jaworski, M. (2013). Decision trees for mining data streams based on the McDiarmid's bound. IEEE Transactions on Knowledge and Data Engineering, 25(6), 1272-1279.

Shaker, A., \& Hüllermeier, E. (2012). IBLStreams: A system for instance-based classification and regression on data streams. Evolving Systems, 3(4), 235-249.

Shi, Z., Wen, Y., Feng, C., \& Zhao, H. (2014a). Drift detection for multi-label data streams based on label grouping and entropy. In 2014 IEEE data mining workshop (ICDMW) (pp. 724-731). IEEE.

Shi, Z., Xue, Y., Wen, Y., \& Cai, G. (2014b). Efficient class incremental learning for multi-label classification of evolving data streams. In 2014 international joint conference on neural networks (IJCNN) (pp. 20932099). IEEE.

Spyromitros-Xioufis, E. (2011). Dealing with concept drift and class imbalance in multi-label stream classification. $\mathrm{PhD}$ thesis, Aristotle University of Thessaloniki.

Struyf, J., \& Džeroski, S. (2006). Constraint based induction of multi-objective regression trees. In Knowledge discovery in inductive databases, LNCS (Vol. 3933, pp. 222-233). Springer.

Triguero, I., \& Vens, C. (2016). Labelling strategies for hierarchical multi-label classification techniques. Pattern Recognition, 56, 170-183.

Tsoumakas, G., \& Katakis, I. (2007). Multi-label classification: An overview. IJDWM, 3(3), 1-13.

Tsoumakas, G., \& Vlahavas, I. (2007). Random k-labelsets: An ensemble method for multilabel classification. In Machine learning: ECML 2007 (pp. 406-417). Springer.

Vens, C., Struyf, J., Schietgat, L., Džeroski, S., \& Blockeel, H. (2008). Decision trees for hierarchical multilabel classification. Machine Learning, 73(2), 185-214.

Xioufis, E. S., Tsoumakas, G., Groves, W., \& Vlahavas, I. P. (2016). Multi-target regression via input space expansion: Treating targets as inputs. Machine Learning, 104(1), 55-98.

Zhang, M. L., \& Zhou, Z. H. (2005). A k-nearest neighbor based algorithm for multi-label classification. In 2005 IEEE granular computing (Vol. 2, pp. 718-721). IEEE. 